\title{
Matching Theory for Channel Allocation in Cognitive Radio Networks
}

\author{
Long $C A O^{1,2}$, Fuqiang $Y A O^{2}$, Hangsheng $Z^{2} H A O^{2}$, Jianzhao ZHANG ${ }^{2}$ \\ ${ }^{1}$ College of Communications Engineering, PLA University of Science and Technology, Houbiaoying Sreet 88, 210014 \\ Nanjing, China \\ ${ }^{2}$ Nanjing Telecommunication Technology Institute, Houbiaoying Sreet 18, 210007 Nanjing, China \\ caolong460@sohu.com,yfq2030@163.com, hassan_zhao@163.com,lgzjz2007@gmail.com
}

Manuscript received December 10, 2015

\begin{abstract}
For a cognitive radio network (CRN) in which a set of secondary users (SUs) competes for a limited number of channels (spectrum resources) belonging to primary users $(P U s)$, the channel allocation is a challenge and dominates the throughput and congestion of the network. In this paper, the channel allocation problem is first formulated as the 0-1 integer programming optimization, with considering the overall utility both of primary system and secondary system. Inspired by matching theory, a many-to-one matching game is used to remodel the channel allocation problem, and the corresponding PU proposing deferred acceptance (PPDA) algorithm is also proposed to yield a stable matching. We compare the performance and computation complexity between these two solutions. Numerical results demonstrate the efficiency and obtain the communication overhead of the proposed scheme.
\end{abstract}

\section{Keywords}

Cognitive radio, channel allocation, optimization, matching theory, stable matching, computation complexity

\section{Introduction}

The rapid proliferation of smartphones, tablets, mobile applications, and other smart devices enable the convenience of our current lifestyle, while spectrum scarcity has become a critical challenge in wireless networks [1]. Cognitive radio (CR) was introduced by Mitola in 1998 as a promising approach to alleviate this status and improve the spectrum utilization efficiency. It allows the unlicensed or secondary users (SUs) to dynamically access the vacant licensed spectrum of primary users (PUs) without the authorization of operators/administrations [2]. To realize cognitive radio networks (CRNs), channel allocation should be adapted according to the varying channel states and requirements, predefined quality of service (QoS) or quality of experience (QoE) [3] of users and other criterion [4-6]. The main purpose of channel allocation is assigning the usable channels or time-frequency chunks to SUs.
The channel allocation problem is difficult because it is a combinatorial integer programming problem of matching SUs to channels. Even in its simplest case, i.e., assign one channel to SUs, it cannot be solved easily or in closed form. This complexity can rapidly increase when the size of the networks growing. At the same time, the global network information and centralized control are required, thus yielding significant additional overhead and complexity.

As one of the distributed channel allocation approach, game theory is utilized to model and analyse the behavior and interactions of users. Considering the inherent selfishness of users, the non-cooperative game supposes that each player is self-interested and wants to maximize its own utility function. The Nash equilibrium of the game is often regarded as the optimal solution. However, formulating the channel allocation problem as a non-cooperative game needs some special constraints and prior knowledges, such as the convexity of the objective functions and the actions of other players, which may not always be satisfied in practice.

Recently, matching theory [8] has emerged as a promising technique for allocation problem, which can overcome some limitations of the existing method [9], [10]. Matching theory is a Nobel Prize winning framework, which is suitable approach to model the interactions between agents in two distinct sets. Most references, such as [11], [12], focus on matching theory in microeconomics. Similarly, the association of channels and SUs can be described by matching theory, one of the first works is presented in [13]. In this work, the channel allocation problem is formulated as a one-to-one matching problem. The transmission rate is considered as the utility function of both SUs and channels. In [14], this theory is applied in CRNs, small cell based networks and device-to-device (D2D) communication these three different areas to demonstrate the usefulness. In [15], matching theory is utilized to tackle the content-cache allocation problem in cache system. Moreover, the cooperative spectrum sharing problem between one PU and SUs is modeled as one-to-one matching game, which optimization is maximizing the utility of secondary network [16]. Apparently, few references concentrate on modelling problem as many-to-one matching. 
In this paper, we focus on the channel allocation mechanism design aiming at maximizing the overall utility or social welfare [17]. The main contributions of this paper are briefly summarized as follows.

1) We model the channel allocation problem as a 0-1 integer programming problem. Differs from the previous work, multiple channels are assigned per SU, and the overall utility function of network considers both secondary system and primary system. Considering the status of primary channel, the secondary system concentrates on the achievable throughput of all SUs, while the primary system focuses on the fee paid by SUs and the achievable throughput of all PUs.

2) We remodel the channel allocation problem as a many-to-one matching [8]. We assume that each primary channel can be assigned to one SU while one SU can be assigned to multiple channels. The maximum number of channels that a SU can access is restricted to its quota. We solve this matching problem by the proposed PU proposing deferred acceptance (PPDA) algorithm, which is a distributed algorithm with lower computation complexity and is optimal to the PUs.

3) Finally, we compare the proposed algorithm with three types of maximal utility mechanisms (including overall utility, PUs utility and SUs utility) and random matching mechanism. The computation complexity analysis is also given. Moreover, we estimate the additional communication overhead under stable matching game scheme according to the number of PU proposals.

\section{System Model}

We consider a cognitive radio network with a set $\mathcal{S}=\left\{S_{1}, \ldots, S_{K}\right\}$ of SUs pairs and a set of orthogonal channels $C=\left\{C_{1}, \ldots, C_{L}\right\}$ licensed to PUs. The total number of SUs $K=|\mathcal{S}|$, where $|\mathcal{S}|$ is the cardinality of the set $\mathcal{S}$, similarly, the number of channels $L=|C|$. The PUs are the spectrum resource owners and have the thresholds of QoS requirements in their allocated frequency bands. Each PU's channel is allowed for one SU to share, while one SU can access several channels simultaneously. We assume that the maximum number of channels that a user $S_{k}$ can access is $q_{k}$, which is determined by the performance of SUs' transceiver and economic budget. The system model is illustrated in Fig. 1.

We assume that the transmission frame is divided into several portions, and the CRN has dedicated control channels. During the signaling period, the channel state information (CSI) is obtained perfectly, which also keep constant within a frame.

As we mentioned above, SUs can take use of the licensed channels within the power restriction. They must vacate the channels when their transmission power exceeds the maximal value of each channel.

First, we define the channel allocation matrix $\mathbf{A}_{L \times K}$ to describe the matching relationship of SUs and channels with

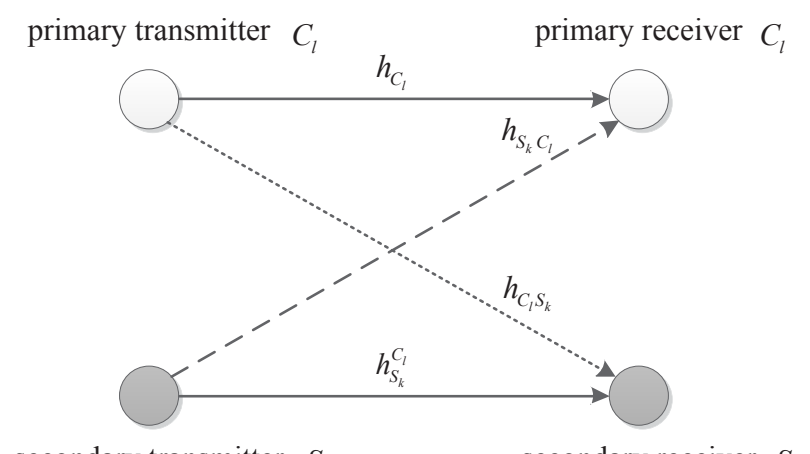

secondary transmitter $S_{k}$

secondary receiver $S_{k}$

Fig. 1. System model.

the $(l, k)$ th element $x_{l k} \in\{0,1\}$, where $l \in\{1, \ldots, L\}$ and $k \in\{1, \ldots, K\}$. If the $l$ th channel is allocated to the $k$ th $\mathrm{SU}$, $x_{l k}=1$, otherwise $x_{l k}=0$. Due to the fact that each channel can be only allocated to one SU, then we obtain the constrain that

$$
\sum_{k, S_{k} \in \mathcal{S}} x_{l k} \leq 1 .
$$

In addition, since user $S_{k}$ can access at most $q_{k}$ channels simultaneously, we have another constrain, i.e.,

$$
\sum_{l, C_{l} \in C} x_{l k} \leq q_{k} \text {. }
$$

\subsection{Utility Function of the Secondary System}

To enhance the flexibility of spectrum access, we introduce the underlay and overlay schemes [5]. Considering these two spectrum access strategies, the average achievable throughput in bit/s/Hz of user $S_{k}$ on channel $C_{l}$ can be formulated as [7]

$$
\begin{aligned}
u_{S_{k}}\left(C_{l}\right) & =P_{0}^{C_{l}} \log _{2}\left(1+\frac{P_{S_{k}}\left|h_{S_{k}}\right|^{2}}{\sigma^{2}}\right) \\
& +\left(1-P_{0}^{C_{l}}\right) \log _{2}\left(1+\frac{P_{S_{k}}\left|h_{S_{k}}\right|^{2}}{\sigma^{2}+P_{C_{l}}\left|h_{C_{l} S_{k}}\right|^{2}}\right)
\end{aligned}
$$

where the first term in the above summation is the throughput when the channel $C_{l}$ is vacant and the second term is the throughput when the channel is occupied, i.e., SU transmission with the PU simultaneously under a tolerable power. $P_{S_{k}}$ and $P_{C_{l}}$ are the transmission power of SU $S_{k}$ and the one of PU working on the channel $C_{l}$, respectively. $h_{S_{k}}^{C_{l}}$ is the channel gain between secondary transmitter and its corresponding receiver on channel $C_{l}, h_{C_{l} S_{k}}$ is the channel gain between primary transmitter and secondary receiver of $S_{k}, \sigma^{2}$ is the noise variance, and $P_{0}^{C_{l}}$ is the probability that the channel is vacant, which is expressed in (4). We assume the status of channels follow a Markov process, which change between vacant and occupied. Let $\alpha_{C_{l}}$ and $\beta_{C_{l}}$ be the probability of transferring from vacant to occupied and transferring from occupied to vacant of channel $C_{l}$, respectively. Then the probability that this channel is vacant can be formulated as

$$
P_{0}^{C_{l}}=\frac{\beta_{C_{l}}}{\alpha_{C_{l}}+\beta_{C_{l}}} .
$$


We assume user $S_{k}$ occupies the channel subset $\mathcal{B}_{k}=$ $\left\{C_{l}: x_{l k}=1\right\} \subseteq C$. Hence, his utility function in this CRN can be represented by the total throughput, i.e.,

$$
U_{S_{k}}\left(\mathcal{B}_{k}\right)=\sum_{l, C_{l} \in C} x_{l k} u_{S_{k}}\left(C_{l}\right)
$$

where $x_{l k}$ is the element of matrix $\mathbf{A}$ which satisfies the constraints (1) and (2), and $u_{S_{k}}\left(C_{l}\right)$ is defined previously in (3). We utilize $u_{S_{k}}(\emptyset)=0$ to denote user $S_{k}$ does not access any channel.

\subsection{Utility Function of the Primary System}

Apparently, the channel $C_{l}$ prefers user $S_{k}$ which will pay higher fee for using, and the achievable throughput of PU on this channel decreases in interference power $P_{S_{k}}\left|h_{S_{k} C_{l}}\right|^{2}$, where $h_{S_{k} C_{l}}$ is the channel gain between secondary transmitter $S_{k}$ and the primary receiver on $C_{l}$. Accordingly, the utility function of channel $C_{l}$ can be formulated as

$$
U_{C_{l}}\left(S_{k}\right)=\rho_{S_{k}}{ }^{x_{l k}} \log _{2}\left(1+\frac{P_{C_{l}}\left|h_{C_{l}}\right|^{2}}{\sigma^{2}+x_{l k} P_{S_{k}}\left|h_{S_{k} C_{l}}\right|^{2}}\right)
$$

where $\rho_{S_{k}}{ }^{x_{l k}} \geq 1$ is the fee paid by user $S_{k}$, and $\rho_{S_{k}}{ }^{x_{l k}}=1$ iff there is no SU active on this channel.

\subsection{Centralized Optimal Solution}

The overall utility of this network is the weighted summation of all SUs' and PUs' utilities, which describes the interest of a network operator/ administration. In economics, this function is also called social welfare [17], and can be represented by

$$
W=\lambda \sum_{k, S_{k} \in \mathcal{S}} U_{S_{k}}\left(\mathcal{B}_{k}\right)+(1-\lambda) \sum_{l, C_{l} \in C} U_{C_{l}}\left(S_{k}\right)
$$

where $\lambda$ is a weighted coefficient which can be utilized to increase the priority of one objective to the other, and $\lambda \in[0,1]$. If $\lambda$ is close to zero, the network pays more attention to primary system performance, while if $\lambda$ is close to one, the secondary system performance is prioritized. In this way, the overall utility will not be dominated by the utility of either side of the users. Hence, the channel allocation problem is considered to maximize the overall utility, which is formulated as

$$
\text { P1: } \begin{aligned}
\max _{\mathbf{A}} & \lambda \sum_{S_{k} \in \mathcal{S}} U_{S_{k}}\left(\mathcal{B}_{k}\right)+(1-\lambda) \sum_{C_{l} \in C} U_{C_{l}}\left(S_{k}\right), \\
\text { s.t. } \quad & x_{l k} \in\{0,1\}, l \leq|C|, k \leq|\mathcal{S}|, \\
& \sum_{S_{k} \in \mathcal{S}} x_{l k} \leq 1, \forall l \in C, \\
& \sum_{C_{l} \in C} x_{l k} \leq q_{k}, \forall k \in \mathcal{S} .
\end{aligned}
$$

There are three constraints in the above optimization problem. Constraint $(\mathrm{C} 1)$ ensures that the status of channels only includes two cases: vacant and occupied. Constraint (C2) guarantees per channel can be allocated to one SU. Constraint (C3) means a SU $S_{k}$ can access multiple channels and the maximum number is $q_{k}$.

We note that $\mathbf{P 1}$ is a $0-1$ integer programming problem with exponential computation complexity, which is N-P hard, and the complexity will increase with the network size. The classical Hungarian algorithm can be applied to solve this problem in centralized manner [18], however, a coordinator is needed to perform the calculation and dictate the channel assignment. Furthermore, some novel programs, such as MATLAB optimization tool or CPLEX developed by IBM [19] also can be utilized to solve this problem automatically. However, we will propose a distributed solution which can achieve a similar performance with lower complexity in next section.

The results, which are actually a centralized solution, will be used to compare with the stable matching algorithm in Sec. 4.

\section{Problem Formulation}

To solve the proposed channel allocation problem, we propose a novel approach based on matching theory. Matching theory originally stems from economics, which is a suitable mathematical framework to analyze and optimize the allocation problem among users and spectrum resources [9]. The merit of matching theory lies in it can provide a distributed solution with tractable computation complexity.

In essence, a matching game is a two-sided assignment problem between two disjoint sets of agents, in which each individual of a set has preferences over the individuals of the opposite set [8]. An individual's preferences represent how a player would choose among different alternatives, if one player was faced with a choice. Economists customarily assume the individual preference is complete ordering and transitive, and using $>_{m}$ as the preference relation of player $m$. For example, $b>_{m} a$ denotes that player $m$ prefers $b$ to $a$. In our application context, preferences associate with the values of utility function.

\subsection{Channel Allocation as a Matching Game}

We first present the fundamental definitions by extending some notations and definitions from previous works in [20], [21], which are applicable to channel allocation in CRN.

Definition 1. A many-to-one matching $\mu$ is a mapping from the set $\mathcal{S} \cup C$ into the set of all subsets of $\mathcal{S} \cup C$ such that for every $S_{k} \in \mathcal{S}$ and $C_{l} \in C$ :

1) $\left|\mu\left(C_{l}\right)\right|=1$ for every channel $C_{l}$ and $\mu\left(C_{l}\right)=C_{l}$ if $\mu\left(C_{l}\right) \notin \mathcal{S}$; 
2) $\left|\mu\left(S_{k}\right)\right|=q_{k}$ for every SU $S_{k}$, and if the number of channels in $\mu\left(S_{k}\right)$, say $r$, is less than $q_{k}$, then $\mu\left(S_{k}\right)$ contains $q_{k}-r$ copies of $S_{k}$;

3) $\mu\left(C_{l}\right)=S_{k}$ if and only if $C_{l} \in \mu\left(S_{k}\right)$.

In our scenario, the channel allocation problem is denoted by the tuple $\left(\mathcal{S}, \mathcal{C}, P,\left\{q_{k}\right\}_{S_{k} \in \mathcal{S}}\right)$, which consists of the set of SUs $\mathcal{S}$, the set of channel resources $C$, the set of preference lists $P=\left\{\left\{U_{S_{k}}\right\}_{S_{k} \in \mathcal{S}},\left\{U_{C_{l}}\right\}_{C_{l} \in C}\right\}$ which is generated according to the utilities of the SUs and PUs given in (5) and (6). The quotas $\left\{q_{k}\right\}_{S_{k} \in \mathcal{S}}$ are associated with SUs. The above definitions imply that the outcome matches the individuals on one side to those on the other side. The propositions 1) and 2) satisfy the constraints (C2) and (C3), respectively.

As we mentioned above, the solution of matching game is stability concept instead of optimality. Next we will introduce related definition of stability.

Definition 2. A matching $\mu$ is individually rational if there exists neither the channel resource is unacceptable to the SU nor the SU is unacceptable to the spectrum resource.

Such a matching is also said to be un-blocked by any unhappy agent.

Definition 3. A matching $\mu$ is blocked by the SU-channel pair $\left(S_{k}, C_{l}\right)$ if $\mu\left(C_{l}\right) \neq S_{k}$ and $S_{k}>_{C_{l}} \mu\left(C_{l}\right)$ and $C_{l}>_{S_{k}} \delta$ for some $\delta$ in $\mu\left(C_{l}\right)$.

In a nutshell, the pair will be said together to block a matching $\mu$ if they are not matched to one another at $\mu$, but would both prefer to be matched to one another than to (one of) their present assignments.

Definition 4. A matching $\mu$ is stable if it is not blocked by any individual agent or any SU-channel pair.

So far, the basic notations and definitions of matching theory have already introduced, in the next subsection we propose an efficient algorithm to solve the matching game, which can obtain stable results.

\subsection{Proposed Algorithm}

In this subsection, we modify the firm proposing deferred acceptance algorithm in [22] to solve the channel allocation problem, which can be extended from the one-to-one stable matching situation. The firm proposing deferred acceptance algorithm was proposed by Gale and Shapley in 1962 and has solved many problems such as National Resident/ Hospital program, college admission [23], roommate market and so on [11]. The weak-optimal channel allocation mechanism that can maximize the overall utility with lower computation complexity is obtained by this algorithm.

In original firm proposing deferred acceptance algorithm, firms first propose to their best choice workers according to the preferences and quotas. Then each worker rejects any unacceptable proposals and "holds" the most preferred if more than one acceptable proposal is received. If no pro- posals are rejected, match each worker to the firm whose proposal he is "holding" and terminate the procedure. The proposing goes on until all workers got accepted or all firms are full.

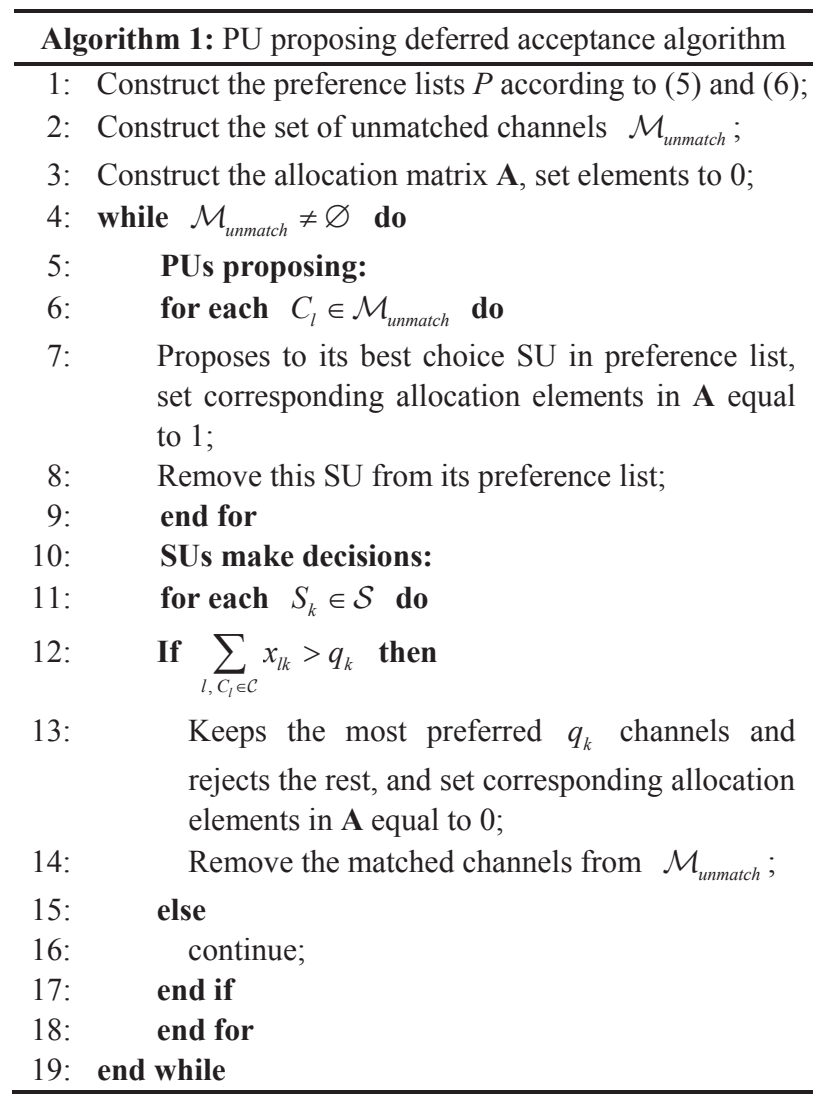

Similarly, we map the orthogonal channels to be the workers, and the SUs to be the firms, since each channel is allocated to one SU while each SU can hold multiple channels. The PPDA algorithm described in Algorithm 1 can be applied to solve $\mathbf{P 1}$ considering the characteristics of channel allocation. We assume the CRN focuses much more attention on the performance of the primary system, i.e., $\lambda$ in (7) is small than 0.5. And we mentioned above that the deferred acceptance algorithm is weak optimal for the proposers, hence, PUs need act as the proposers in our scenario. Firstly, CRN executes the initialization of Algorithm 1 (Line 1 to 3 ), including constructs the needed matrixes and preference lists. Moreover, all SUs must have the knowledge of channels' activities and the interference $P_{C_{l}}\left|h_{C_{l} S_{k}}\right|^{2}$, and all PUs require the knowledge of the fee paid by SUs and the interference $P_{S_{k}}\left|h_{S_{k} C_{l}}\right|^{2}$, the interference values can be calculated during channel estimation period. Secondly, PUs propose to most preferred channels according to their preference lists (Line 5 to 9). Then SUs decide whether accept these proposals based on their preferences and quotas (Line 10 to 18 ), then feed back the results to corresponding PUs. Finally, the matching process ends when all primary channels are allocated, and this status is a PU-optimal stable matching which is weak Pareto optimal. Furthermore, while the status of network changes, Algorithm 1 will be implemented once more. 


\section{Simulation Results and Analysis}

Considering a $\mathrm{CRN}$ in a $300 \mathrm{~m} \times 300 \mathrm{~m}$ square area to numerically evaluate the proposed algorithm, the number of orthogonal channels $L=10$ and the number of SUs $K$ ranges from 1 to 6. Each SU's access ability, i.e. the quotas $q_{k}$ is assumed equal to 2 . The distribution of users is shown in Fig. 2, which is generated randomly, with solid dots donating transmitters, hollow dots representing receivers. The PU's transceivers are in red color, while the SU's transceivers are in blue color. We simply assume the transmission power of PUs is set to $5 \mathrm{~W}$, and the distance between each PU's transmitter and relative PU's receiver is identical for all as $100 \mathrm{~m}$. Similarly, the transmission power of SUs is set to $1 \mathrm{~W}$, and the distance is equal to $80 \mathrm{~m}$. The wireless fading channels are independent identically distributed (i.i.d.) and Rayleigh distribution with the coefficient $\lambda_{0}=1$, the pathloss fading component $\kappa=4$. The setting of rest parameters are provided in Tab. 1 .

In simulations, we fix the number of channels and compare stable matching algorithm with the maximal social welfare, maximal SUs utility and maximal PUs utility. These three allocation mechanisms are under different number of SUs, which the optimization objective is to maximize the overall utility, SUs utility and PUs utility respectively. In addition, we use the random matching mechanism as the benchmark to compare with the above mechanism. In the random allocation mechanisms, we assign channels randomly to SUs under the constraints on $q_{k}$, and the simulation results are the average of $10^{6}$ times.

Figure 3 shows the social welfare of the network while the number of SUs ranges from 1 to 6 . Apparently, the stable matching mechanism and maximal utility mechanism achieve higher value of social welfare than random matching mechanism. In our scenario, the PUs utility occupies a predominant position in overall utility function. Hence, the maximal PUs utility mechanism almost achieves the same value of maximal social welfare. The stable matching mechanism performs slightly worse than the maximal social welfare, for example, when the number of SUs $K=3$, the social welfare are equal to 2.93 and 2.66, respectively (roughly $9.2 \%$ worse than the maximal social welfare method). Moreover, we observe that as the number of SUs increasing, the gap between these two methods decreases. However, the first three mechanisms belong to 0-1 integer programming optimization, which have higher computation complexity and increase exponentially over the network size. While the complexity of stable matching algorithm is $O(K \times L)$, where $K \times L$ is the number of possible SU-channel pairs.

\begin{tabular}{|c|c|}
\hline Parameter & Value \\
\hline \hline$\lambda$ & 0.4 \\
\hline$\rho_{S_{k}}$ & 2 \\
\hline$\alpha$ & $1 / 3$ \\
\hline$\beta$ & $1 / 2$ \\
\hline
\end{tabular}

Tab. 1. The setting of parameters.

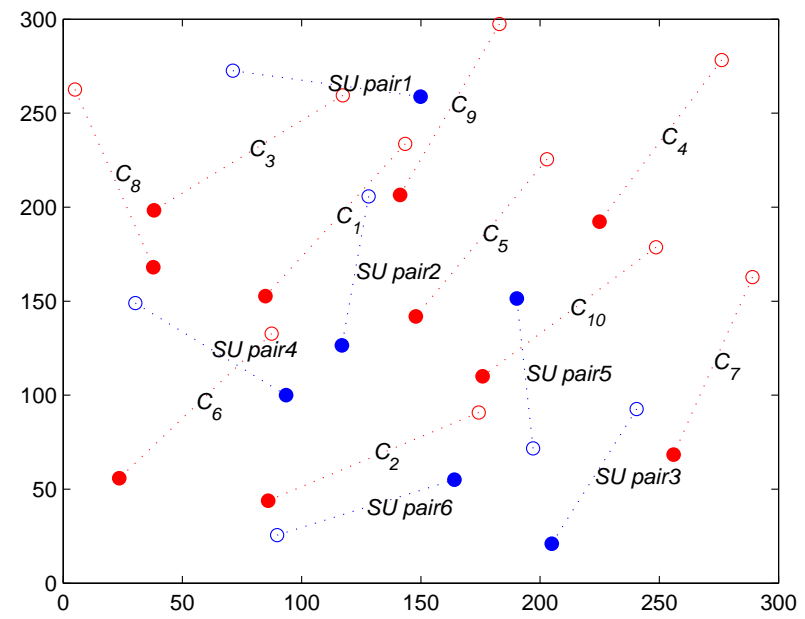

Fig. 2. Distribution of users.

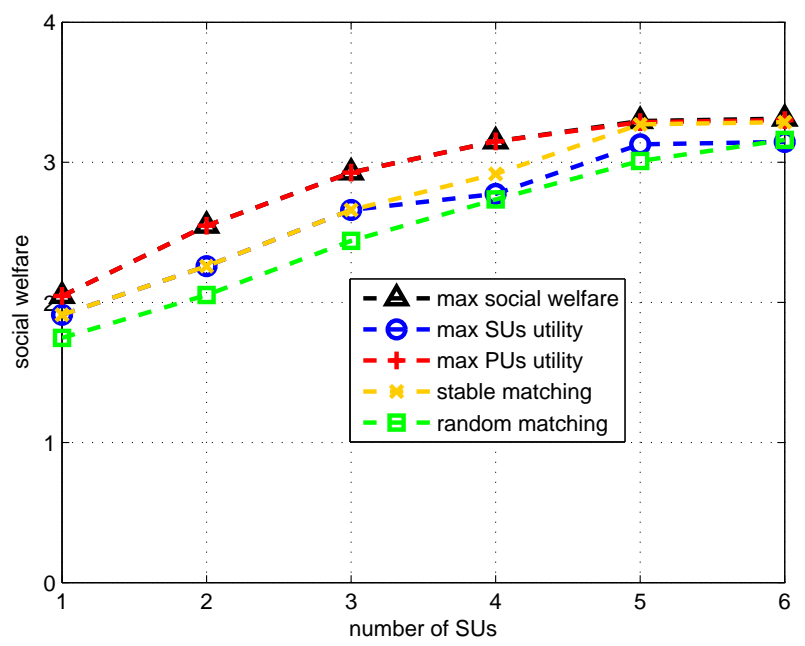

Fig. 3. The social welfare of the network vs. SU number.

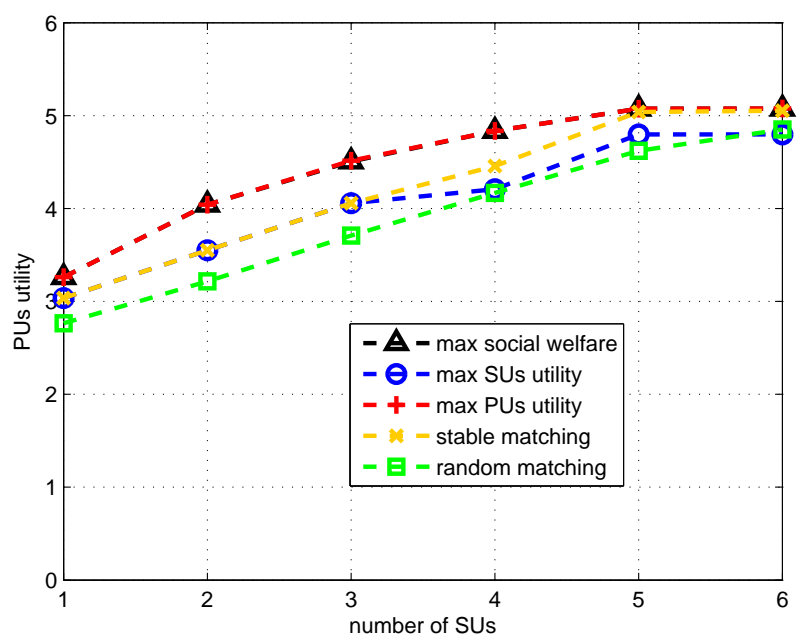

Fig. 4. The utility of PUs vs. SU number.

Figure 4 shows the utility of PUs while the number of SUs varies. The curves in this figure are similar to Fig. 3 due to the significant situation of PUs utility in (7). With the increasing of the SUs number, stable matching mechanism can achieve better utility level, even is equivalent to the maximal social utility. 


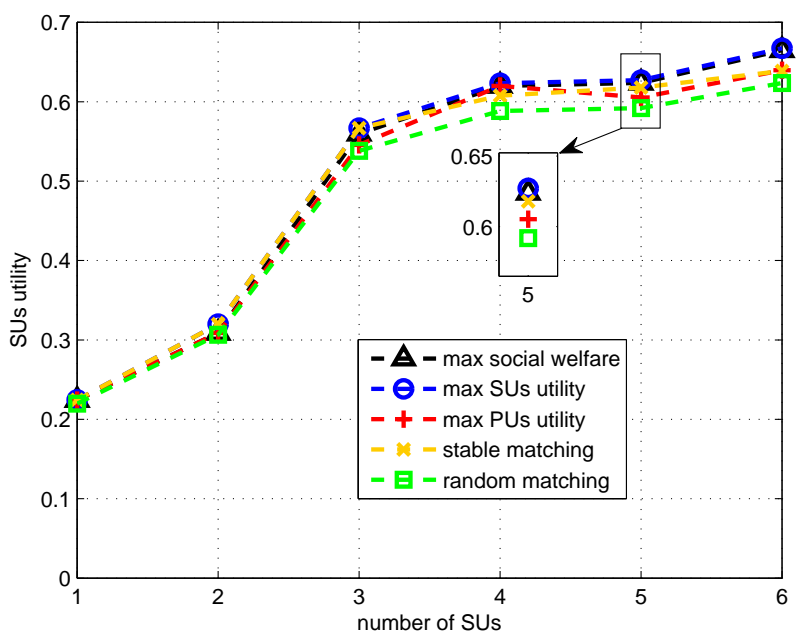

Fig. 5. The utility of SUs vs. SU number.

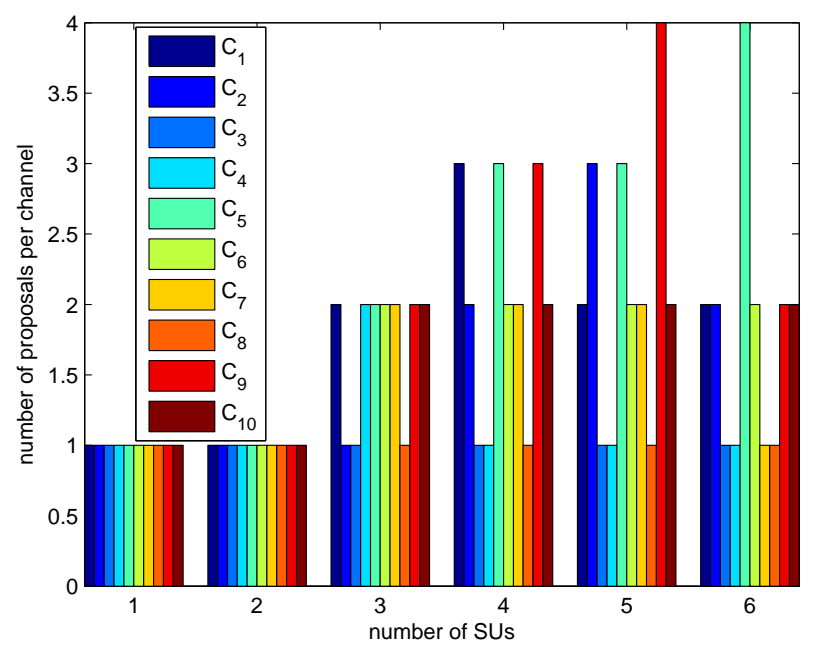

Fig. 6. Number of proposals per channel.

In Fig. 5, the first four mechanisms achieve less difference in SUs utility. Especially, the values of stable matching and maximal PUs utility are almost the same since the PPDA algorithm is PU-optimal, which means the PUs will benefit much more from matching.

Figure 6 shows the number of proposals per channel under stable matching game scheme for increasing number of SUs. Obviously, while the number of SUs is much smaller than the available channels, i.e. $K \leq 3$, each channel can get the matched SU by proposing only one or two times. However, as the number of SUs increases, channels must raise more proposals to achieve stable because finding a preferred SU becomes more competitive. It can be observed that channel $C_{3}$ and $C_{8}$ propose only once all the time due to the distinctive preferences of these two channels. By calculating the preferences of this matching game, we obtain that the first choice of channel $C_{3}$ and $C_{8}$ is SU $S_{3}$, and the preference of $\mathrm{SU} S_{3}$ is $\left\{C_{8}, C_{3}, \ldots\right\}$. Hence, we can conclude that $\mathrm{SU} S_{3}$ will engage the first proposals from channel $C_{3}$ and $C_{8}$.

Figure 7 plots the average number of PU proposals for increasing number of SUs. The average number of proposals increases for larger $K$. For $\sum_{k=1}^{K} q_{k}>L$, i.e. $K \geq 6$ in our

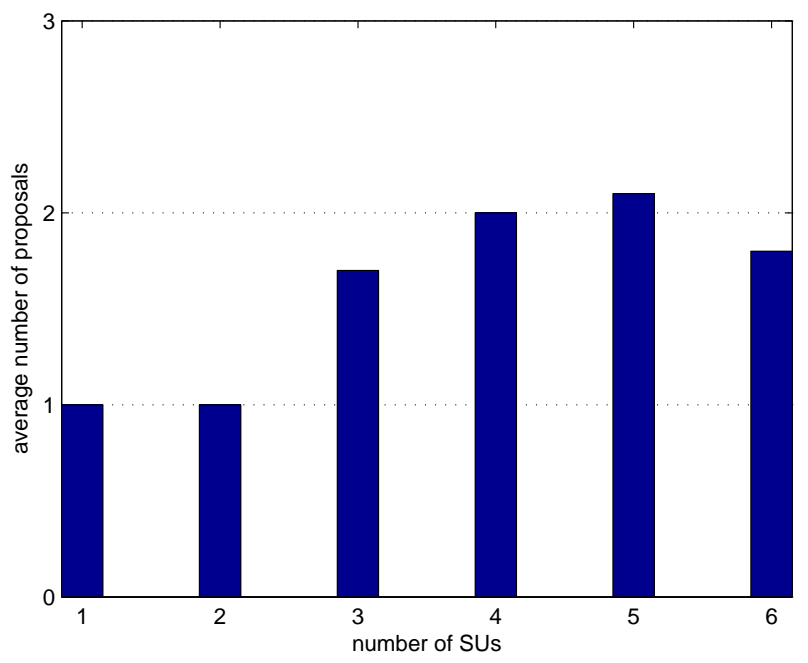

Fig. 7. Average number of proposals vs. SU number.

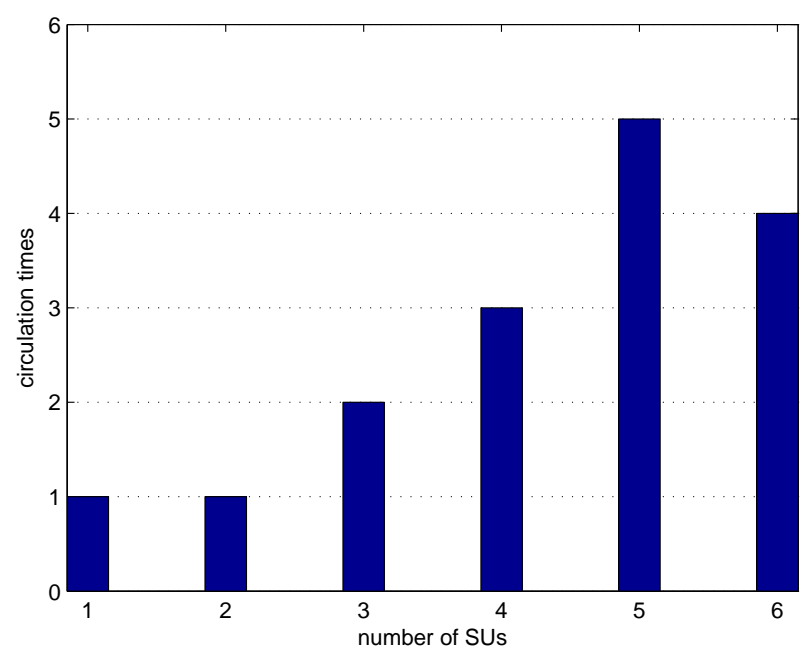

Fig. 8. Circulation times vs. SU number.

simulation setting, where channel resources are unable to satisfy the SUs' requirements, the average number of proposals decreases. Note that there exist some SUs only matched with one channel, and channels have more SUs to choose in such case. Hence, a few average number of PU proposals is needed for our PPDA algorithm. Furthermore, we can estimate the additional communication overhead according to Fig. 6 and Fig. 7 due to the adoption of stable matching scheme. It is reasonable to assume that $\mathrm{PU} C_{l}$ proposes through $M$ bit message to its preferred SU, and the preferred SU responds this proposal via $N$ bit message. Hence the communication overhead can be calculated as

$(M+N) \times L \times$ avg. number of proposals per channel.

Figure 8 shows the circulation times of PPDA algorithm, and we consider line 5 to 18 as one time. The simulation results are similar to Fig. 7 since the lower average number of PU proposals, the fewer circulation times should be implemented. 


\section{Conclusion}

In this paper, the matching theory is applied to model and address the channel allocation problem. The overall utility considers the utility of both secondary system and primary system. The 0-1 integer linear programming algorithms optimize the channel allocation problem of three different goals. Then we model this problem as a stable matching game once more, in addition, the corresponding PPDA algorithm is proposed to reach the stable states with lower computation complexity. Simulation results verify the efficiency of this mechanism and present the possible communication overhead.

\section{Acknowledgments}

This research was supported by the Natural Science Foundation of China under grants. 61471395, 61301161, and the Natural Science Foundation of Jiangsu Province under grants. BK20141070, BK20161125.

\section{References}

[1] HUAWEI. 5G a technology vision. [Online] Cited 2015-12-10. Available at: http://www.huawei.com/ilink/en/download/HW_314849

[2] MITOLA, J. Cognitive radio (Licentiate proposal). Stockholm (Sweden), KTH, 1998.

[3] MITOLA, J., GUERCI, J., REED, J., et al. Accelerating 5G QoE via public-private spectrum sharing. IEEE Communications Magazine, May 2014, vol. 52, no. 5, p. 77-85. ISSN: 0163-6804. DOI: $10.1109 /$ MCOM.2014.6815896

[4] AHMAD, A., AHMAD, S., REHMANI, M. H., et al. A survey on radio resource allocation in cognitive radio sensor networks. IEEE Communications Surveys \& Tutorials, 2015, vol. 17, no. 2, p. 888-917. DOI: 10.1109/COMST.2015.2401597

[5] TSIROPOUlos, G. I., DOBRE, O. A., AHMED, M. H., et al. Radio resource allocation techniques for efficient spectrum access in cognitive radio networks. IEEE Communications Surveys \& Tutorials, 2016, vol. 18, no. 1, p. 824-847. DOI: 10.1109/COMST.2014.2362796

[6] BAYAT, S., LOUIE, R. H. Y., HAN, Z., et al. Distributed user association and femtocell allocation in heterogeneous wireless networks. IEEE Communications Surveys \& Tutorials, 2014, vol. 62, no. 8, p. 3027-3043. DOI: 10.1109/TCOMM.2014.2339313

[7] LIANG, Y.-C., ZENG, Y., PEH, E. C. Y., et al. Sensingthroughput tradeoff for cognitive radio networks. IEEE Transactions on Wireless Communications, 2008, vol. 7, no. 4, p. 1326-1337. DOI: 10.1109/TWC.2008.060869

[8] ROTH, A., SOTOMAYOR, M. A. O. Two-Sided Matching: A Study in Game-Theoretic Modeling and Analysis. Cambridge University Press, 1992. ISBN: 9780521390156

[9] VASSAKI, S., POULAKIS, M. I., PANAGOPOUlOS, A. D. Spectrum leasing in cognitive radio networks: A matching theory approach. In Proceedings of the the 81st IEEE Vehicular Technology Conference. Glasgow (UK), May 2015, p. 1-5. DOI: 10.1109/VTCSpring.2015.7146101
[10] YAFFE, Y., LESHEM, A., ZEHAVI, E. Stable matching for channel access control in cognitive radio systems. In Proceedings of the International Workshop on Cognitive Information Processing (CIP). Jun. 2010, p. 470-475. DOI: 10.1109/CIP.2010.5604115

[11] MANLOVE, D. F. Algorithmics of Matching Under Preferences. World Scientific, 2013. ISBN: 9789814425247

[12] IRVING, R. W., LEATHER, P., GUSFIELD, D. An efficient algorithm for the optimal stable marriage. Journal of the Association for Computing Machinery, 1987, vol. 34, no. 3, p. 532-543. DOI: $10.1145 / 28869.28871$

[13] LESHEM, A., ZEHAVI, E., YAFFE, Y. Multichannel opportunistic carrier sensing for stable channel access control in cognitive radio systems. IEEE Journal on Selected Areas in Communications, 2012, vol. 30, no. 1, p. 82-95. DOI: 10.1109/JSAC.2012.120108

[14] GU, Y., SAAD, W., BENNIS, M., et al. Matching theory for future wireless networks: fundamentals and applications. IEEE Communications Magazine, 2015, vol. 53, no. 5, p. 52-59. DOI: 10.1109/MCOM.2015.7105641

[15] GU, Y., ZHANG, Y., PAN, M., et al. Student admission matching based content-cache allocation. In Proceedings of the IEEE Wireless Communications and Networking Conference. New Orleans (USA), Mar. 2015, p. 2179-2184. DOI: 10.1109/WCNC.2015.7127805

[16] NAMVAR, N., AFGHAN, F. Spectrum sharing in cooperative cognitive radio networks: A matching game framework. In Proceedings of the IEEE the 49th Annual Conference on Information Sciences and Systems (CISS). Baltimore (USA), Mar. 2015, p. 1-5. DOI: $10.1109 /$ CISS.2015.7086843

[17] ARROW, K. J. Social Choice and Individual Values. 2nd ed. Yale University Press, 1963. ISBN: 9780300013634

[18] PAPADIMITROU, C., STEIGLITZ, K. Combinatorial Optimization: Algorithms and Complexity. Dover Press, 1998. ISBN: 9780486402581

[19] IBM. Cplex Optimizer. [Online] Cited 2015-12-10. Available at: http://www-01.ibm.com/software/commerce/optimization/cplexoptimizer/.

[20] JORSWIECK, E. Stable matchings for resource allocation in wireless networks. In Proceedings of the 17th International Conference on Digital Signal Processing (DSP). Corfu, Jul. 2011, p. 1-8. DOI: 10.1109/ICDSP.2011.6004983

[21] BAYAT, S., LOUIE, R., HAN, Z., et al. Physical-layer security in distributed wireless networks using matching theory. IEEE Transactions on Information Forensics and Security, 2013, vol. 8, no. 5, p. 717-732. DOI: 10.1109/TIFS.2013.2251335

[22] ROTH, A. E. The evolution of the labor market for medical interns and residents: A case study in game theory. Journal of Political Economy, 1984, vol. 92, no. 6, p. 991-1016. DOI: $10.1086 / 261272$

[23] DALE, D., SHAPLEY, L. S. College admissions and the stability of marriage. The American Mathematical Monthly, 1962, vol. 69, no. 1, p. 9-15. DOI: $10.2307 / 2312726$

\section{About the Authors...}

Long CAO received the M.S. degree in Communications and Information Systems from PLA University of Science and Technology, Nanjing, China in 2013. He is currently pursuing for the Ph.D. degree at the College of Communications Engineering, PLA University of Science and Technology. His current research interests include cognitive radio networks, spectrum management and resource allocation. 
Fuqiang YAO (corresponding author) received the M.S. degree and Ph.D. degree in Communications and Electronic Systems both from Xidian University, Xi'an, China in 1990 and 1993 respectively. Currently, he is a professor at the Nanjing Telecommunication Technology Institute, China. His research interests include wireless communications, spectrum management and communication anti-jamming.

Hangsheng ZHAO received the M.S. degree and Ph.D. degree both from PLA University of Science and Technology, in 1991 and 1999 respectively. From March 2000 to February 2002, he was a post doctor at the Shanghai Jiao Tong University, China. From September 2011 to September 2012, he was a senior visiting scholar at the University of Waterloo, Canada. Currently, he is a professor at the Nanjing Telecommunication Technology Institute, China. His research interests include wireless communications and spectrum management.

Jianzhao ZHANG received the B.S. degree and Ph.D. degree both from PLA University of Science and Technology, in 2007 and 2012 respectively. Since 2012, he has been with the Nanjing Telecommunication Technology Institute, China. His research interests include Ad Hoc networks and spectrum management. 